RECyT

Año 22 / No 33 / 2020 / 100-107

\title{
Efectos de los microplásticos en el medio ambiente: Un macroproblema emergente
}

\section{Efects of microplastics on the environment: an emerging macroproblem \\ Efeitos dos microplásticos no meio ambiente: Um macroproblema emergente}

\author{
Lisandro D. Giraldez Alvarez ${ }^{1,}{ }^{*}$, Fernanda Braz De Jesus ${ }^{1}$, Ana P. Lacerda Costa ${ }^{1}$, Letícia

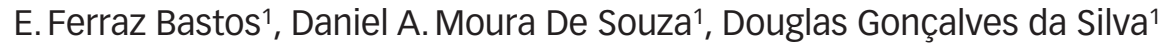

1- Laboratório de Química Analítica e Ambiental. Departamento de Ciências Naturais (DCN). Universidade Estadual do Sudoeste da Bahia (UESB). ESTRADA DO BEM QUERER, KM 4, BAIRRO UNIVERSITÁRIO, VITORIA DA CONQUISTA, Bahia, CEP: 45031-300. Brasil.

*E-mail: giraldezli@gmail.com

\section{Resumen}

Recibido el 19 de noviembre de 2019, Aprobado el 16 de marzo de 2020.

\begin{abstract}
Como material liviano, duradero, versátil y potencialmente transparente, los plásticos son ideales para una variedad de aplicaciones. Sin embargo, se producen alrededor de 300 millones de toneladas de plástico por año, de las cuales 13 millones llegan a ríos y océanos lo que puede causar daños irreparables a esos ecosistemas y afectar organismos con capacidad limitada de adaptación. Se estima que hasta el momento se han producido 8300 millones de toneladas métricas (TM) de plástico, de las cuales 6300 TM se han transformado en desechos a partir de 2015, y solo el $9 \%$ fueron reciclados. El riesgo que pueden representar los microplásticos (MP's) para la salud humana aún no es un problema definido, pero existen numerosas evidencias de los impactos negativos del plástico y sus desechos, los microplásticos y nanoplásticos, ingeridos por organismos de diversos ecosistemas. Por lo tanto, el objetivo de este trabajo fue llevar a cabo una investigación bibliográfica sobre el estado actual del conocimiento sobre los microplásticos y sus impactos en el medio ambiente, especialmente en ambientes acuáticos.
\end{abstract}

Palabras clave: Microplásticos; Medio ambiente; Contaminación acuática.

\section{Abstract}

As a lightweight, durable, versatile and potentially transparent material, plastics are ideal for a variety of applications. However, around 300 million tons of plastic are produced per year, of which 13 million reach rivers and oceans causing irreparable damage to this ecosystem and affecting organisms with limited adaptation capacity. It is estimated that 8300 million metric tons (MT) of plastic has been produced. From 2015 onwards, 6300 MT has become waste and only $9 \%$ of it has been recycled. The risk that microplastics (MPś) can represent for human health is not yet a definite problem, but there is numerous evidence of the negative impacts of plastic and their wastes, microplastics and nanoplastics, ingested by organisms from diverse ecosystems. Therefore, the objective of this work was to carry out a bibliographic investigation on the current state of knowledge about microplastics and their environmental impacts, especially in the aquatic ones.

Keywords: Microplastic; Environment; Aquatic pollution.

\section{Resumo}

Por tratar-se de um material leve, resistente, versátil e potencialmente transparente, os plásticos se tornam ideais para uma diversidade de aplicações. Porém, cerca de 300 milhões de toneladas de plástico são produzidos por ano, dos quais 13 milhões são levados aos rios e oceanos podendo causar danos irreparáveis a esse ecossistema afetando os organismos com capacidade limitada de adaptação. Estima-se que 8300 milhões de toneladas métricas (MT) de plástico foram produzidas até o momento, das quais $6300 \mathrm{MT}$ se tornaram resíduos a partir de 2015, e foram reciclados apenas $9 \%$. Mesmo que os microplásticos (MP's) estejam onipresentes nos oceanos, o risco que podem causar à saúde humana ainda não é uma questão definida. As evidências dos impactos negativos do plástico e seus detritos microplásticos e nanoplástico ingerido pelos organismos de diversos ecossistemas, são fornecidos constantemente pela comunidade científica. Por isso, o objetivo deste trabalho foi realizar uma investigação bibliográfica sobre o estado atual do conhecimento sobre os microplásticos e os seus impactos sobre o meio ambiente, principalmente nos ambientes aquáticos.

Palavras-chave: Microplásticos; Médio ambiente; Contaminação aquática

RECYT / Año 22 / N 33 / 2020 


\section{Introdução}

A produção de materiais pelo ser humano na sua procura pela praticidade no dia a dia ocasiona, muitas vezes, vários problemas relacionados à saúde em geral e à preservação do meio ambiente. Nas últimas décadas a produção de plástico cresceu radicalmente e, como consequência, o seu acúmulo e contaminação do meio ambiente como foi observado, por exemplo, no estuário de Goiana, Brasil, e no Delta Atrato, Colômbia [1] [2].

A compreensão das vias de poluição e dos impactos decorrentes nos ecossistemas é importante não apenas para um melhor entendimento das respostas negativas, mas também para formular medidas de prevenção [3]. Nesse sentido, um problema que vem sendo discutido atualmente são os resíduos de plásticos nos ambientes aquáticos, por trazerem consequências adversas aos animais marinhos e a saúde humana. A crescente contaminação de nossos oceanos com detritos plásticos é um problema de grande preocupação ambiental. Recentemente, estimou-se que 8300 milhões de toneladas métricas (MT) de plástico foram produzidas até o momento, das quais $6300 \mathrm{MT}$ se tornaram resíduos a partir de 2015, dos quais foram reciclados apenas $9 \%$ [4].

Para se compreender as fontes e vias de poluição do plástico nos ambientes aquáticos é necessária uma avaliação abrangente sobre os riscos desses contaminantes orgânicos, para se ter uma definição, o entendimento e a quantificação do lançamento desses resíduos, tanto geográfico quanto temporal [5].

Por tratar-se de um material leve, resistente, versátil e potencialmente transparente, os plásticos se tornam ideais para uma diversidade de aplicações. Principalmente quando se leva em conta suas notáveis propriedades de barreira ao oxigênio/umidade, seu baixo custo, a bioinércia e sua leveza, que fazem deles formidáveis materiais para embalagem [6]. Apesar disso, as evidências dos impactos negativos do plástico e seus detritos microplásticos e nanoplástico ingeridos pelos organismos de diversos ecossistemas são fornecidas constantemente pela comunidade científica [7].

O Joint Group of Experts on the Scientific Aspects of Marine Environmental Protection (GESAMP), define microplásticos como "partículas de plástico com menos de 5 $\mathrm{mm}$ de diâmetro, que incluem partículas na faixa de nano-tamanho (1 nm)" [8]. Sem embargo, considerando toda a atenção e pesquisa que existe até o momento, ainda não há um consenso claro sobre uma definição que seja extensa o suficiente para abranger todos os critérios necessários para descrever os 'microplásticos', por isso recentemente foi proposta a seguinte definição para os microplásticos: "Microplásticos são qualquer partícula sólida sintética ou matriz polimérica, com forma regular ou irregular e com tamanho variando de $1 \mu \mathrm{m}$ a $5 \mathrm{~mm}$, de origem primária ou secundária de fabricação e insolúveis em água" [9].
Os microplásticos são de grande ameaça à biota, por serem persistentes e levarem longos períodos para se degradar, devido também ao seu pequeno tamanho, pois podem ser ingeridos por uma grande variedade de organismos, os quais, por sua vez são afetados por conta de sua grande capacidade de absorver e concentrar contaminantes, fator que acarreta vários efeitos [10]. Além disso, os microplásticos apresentam o potencial de absorver contaminantes químicos, o que leva os organismos aquáticos à ingestão de poluentes orgânicos e inorgânicos [11]. Ressalta-se, ainda, que sua abundância continue a aumentar, despertando uma séria preocupação para os seres humanos e a fauna marinha [7].

Embora muitas investigações, incluindo várias revisões críticas sobre MPs no ambiente tenham sido conduzidas, a maioria delas está focada em suas ocorrências no ambiente marinho [12], sendo importante destacar que de 195 países no mundo só 23 estudaram a poluição por plásticos em água doce [13]. Por isso, o objetivo deste trabalho é realizar uma investigação bibliográfica sobre o estado atual do conhecimento sobre os referidos microplásticos e os seus impactos sobre o meio ambiente, principalmente nos ambientes aquáticos.

\section{Efeitos dos microplásticos sobre os organismos marinhos}

O conhecimento de sua distribuição em ambientes remotos como regiões polares e o mar profundo é escasso. Amostras de sedimentos coletadas no observatório Ártico HAUSGARTEN a profundidades entre 2340 e $5570 \mathrm{~m}$ mostraram uma elevada quantidade de microplásticos (42-6595 microplastics $/ \mathrm{kg}$ ) com um tamanho de $\leq 25$ $\mu \mathrm{m}$ em $80 \%$ das amostras. Um total de 18 polímeros diferentes foram encontrados com o polietileno clorado na maior proporção $(38 \%)$, seguido pela poliamida $(22 \%)$ e polipropileno (16\%), indicando que os mares podem ser importantes transportadores desse material [14].

\section{Efeitos sobre peixes}

Diversos são os efeitos negativos da ingestão de micro e nanoplásticos pelos peixes. Análises mostraram que a carga total desses poluentes no trato gastrointestinal de um peixe durante a sua vida é alta, o que consequentemente poderá afetar sua saúde. Observou-se, como efeitos, o bloqueio intestinal, o dano físico, as alterações histopatológicas no intestino, as mudanças de comportamento e do metabolismo lipídico e sus transferência para o fígado [15]. Resultados preocupantes foram obtidos a partir de estudos realizados no estuário do Douro (Portugal) que microplásticos, bem como a abundância e distribuição desses resíduos e larvas de peixes, os quais mostraram que a densidade que MPs superou a de larvas de peixes na maioria das estações e áreas estuarinas onde foram coletadas (na proporção de 1,0 larvas: 1,5MPS). Os microplásticos (MPs $\leq 5 \mathrm{~mm}$ ) são particularmente perigosos para os estágios iniciais da 
vida dos peixes, porque sua ingestão pode induzir bloqueio intestinal, limitando a ingestão de alimentos ou expondo os organismos à contaminação devido à capacidade dos MPs. de absorção de poluentes [16].

\section{Efeitos sobre corais}

Constatou-se que uma espécie de coral de água fria exposta por um período de 5 meses a micro e macroplásticos, apesar de não ter ocasionado a morte, afetou o organismo tanto em termos de captura quanto em taxa de crescimento. Os pólipos de Lophelia pertusa, expostos aos microplásticos, apresentaram um crescimento consideravelmente menor em relação ao controle. Além disso, foi observado o crescimento reduzido do septo sob a exposição microplástica em comparação às condições de controle (307 \pm $360 \mathrm{~mm}$ e $1260 \pm 770 \mathrm{~mm}$, respectivamente). Neste estudo foram analisadas em conjunto, outras espécies, as quais não expressaram alterações significativas. No entanto, considerando-se que algumas espécies podem ser mais afetadas que outras, a poluição plástica pode levar a uma redução da biodiversidade nos ecossistemas onde esses organismos habitam [17]. Seis espécies de corais pétreos de pólipos pertencentes aos gêneros Acropora, Pocillopora e Porites foram expostos a microplásticos (polietileno, tamanho 37-16 $\mu \mathrm{m}$, concentração ca. 4000 partículas $\mathrm{L}^{-1}$ ) durante quatro semanas. Cinco das seis espécies analisadas apresentaram respostas diferentes à exposição, como por exemplo a fixação de microplásticos aos tentáculos ou filamentos mesenteriais, produção de muco, crescimento excessivo ou morte tecidual [18].

Para análise dos efeitos a longo prazo em corais, realizou-se um estudo de microcosmo nos organismos pertencentes aos gêneros Acropora, Porites, Pocillopora e Heliopora. Estes foram expostos, por um período de seis meses, a uma concentração de 200 particulas $\mathrm{L}^{-1}$ microplásticas relacionadas a níveis de poluição. As espécies Acropora muricata e Pocillopora verrucosa mostraram sinais de comprometimento da saúde seis semanas após início da exposição. A taxa de crescimento (mudanças na área da superfície, volume e taxa de calcificação) foi avaliada em análise de varredura 3D e medições de peso, nas quais foi possível observar impactos negativos em $A$. muricata e $H$. coerulea. Na primeira, o crescimento da área superficial foi $33 \%$ menor quando comparado ao grupo controle; a segunda apresentou taxas de calcificação reduzidas em 3\% sob a exposição, mostrando ao longo do tempo a ocorrência da diminuição da força do efeito. Esses dados mostram como a exposição a microplásticos pode ter efeitos negativos em algumas espécies importantes de corais [19].

\section{Efeitos sobre ostras}

Foi possível observar o impacto de microesferas de poliestireno (micro-PS) na fisiologia da ostra do Pacífi- co. Ostras adultas foram expostas experimentalmente ao micro-esferas de poliestireno, por 2 meses durante um ciclo reprodutivo. Após 2 meses, as ostras expostas apresentaram significativas reduções na velocidade espermática (-23\%), no diâmetro (-5\%) e no número de oócitos $(-38 \%)$. Em comparação com os filhos controle, o rendimento das larvas e o desenvolvimento larval dos filhos derivados de pais expostos diminuíram $41 \%$ e $18 \%$, respectivamente [20].

$\mathrm{Na}$ região oriental da Bacia do Levantine (na costa libanesa) foi avaliada, pela primeira vez, a poluição microplástica e os polímeros encontrados mais frequentemente. Neste estudo, foram coletadas em Tripoli, Beirute e Sidon duas espécies de frutos do mar anchovas (Engraulis encrasicolus) e ostras-espinhosas (Spondylus spinosus) regularmente consumidas pela comunidade libanesa, assim como água do mar e sedimentos dos mesmos locais. Os resultados apresentaram diferentes padrões na concentração de MPs: a água de Sidon apresentou elevado grau de contaminação $\left(6,7 \mathrm{MPs} / \mathrm{m}^{3}\right)$ por microplásticos, enquanto em Tripoli a maior contaminação foi observada nos sedimentos (4,68 MPs/g). Foram observados $83,4 \%$ e $86,3 \%$ de microplásticos na biota de anchovas e ostrasespinhosas, respectivamente, mas os índices mais altos de MPs/indivíduo foram vistos nas anchovas e ostras da região de Beirute. Os autores ainda acrescentam que a quantidade de resíduos ingeridos por esses animais pode resultar em potenciais problemas de saúde, já que há alto consumo dessas espécies na região; e atribuem a grande quantidade de microplásticos na Bacia oriental do Mediterrâneo à presença dos aterros costeiros e dos resíduos mal administrados [21].

\section{Efeitos sobre Zooplâncton}

A contaminação por microplásticos do ambiente aquático é considerada um problema crescente. São, portanto, notórios os efeitos que a ingestão de microplásticos pelos animais marinhos - incluindo mexilhões, peixes, aves marinhas e até mesmo zooplânctons - trazem para a biota. Apesar de haver poucas pesquisas relacionados à ingestão dos microplásticos por zooplânctons, através de estudos realizados em laboratório usando a microscopia de fluorescência foi identificado que trece taxones de zooplancton tenían la capacidad de ingerir bolas de poliestireno de tamanhos que varia de 1.7 a $30.6 \mu \mathrm{m}$, con una absorción por parte de los indivíduos que varía según los taxones, la etapa de la vida y el tamaño de las bolas, fato que pode influenciar as funções e a saúde dos indivíduos [22].

\section{Efeitos dos microplásticos em organismos de água doce}

Até agora, a maioria das pesquisas em microplásticos se concentrou em o ambiente marinho como fui apresentado por Bletter et al (2018) mostrado que $87 \%$ dos estudos de poluição por plásticos estão relacionados aos ambientes 
marinhos e apenas $13 \%$ aos sistemas de água doce, com uma taxa de crescimento de aproximadamente 41 vs. 7 artigos por ano, para ambientes marinhos e de água doce, respectivamente [13]. Embora os ambientes de água doce e terrestre sejam reconhecidos como origens e vias de transporte de plásticos para os oceanos, ainda existe uma falta comparativa de conhecimento sobre essas questões ambientais [23].

A ingestão de microplásticos por invertebrados de água doce não foi relatada fora de estudos realizados em laboratório. Sem embargo, em um grande corpo de água urbano alimentado pelo rio Irwell, em Manchester, Reino Unido, foi estudada a ingestão de partículas microplásticas de sedimentos de fundo por Tubifex tubifex. Os sedimentos hospedeiros apresentaram concentrações microplásticas variando de 56 a 2544 partículas/kg, 87\% das partículas microplásticas ingeridas por Tubifex eram microfibras (55 - $4100 \mu \mathrm{m}$ de comprimento), enquanto os $13 \%$ restantes eram fragmentos microplásticos $(50-4500 \mu \mathrm{m}$ de comprimento). Enquanto as microesferas estavam presentes na matriz de sedimentos do hospedeiro, elas não foram detectadas no tecido do verme Tubifex. A concentração média de microplásticos ingeridos foi de $129 \pm 65,4$ partículas tecido/g. Também foi mostrado, nesse estudo, que os vermes Tubifex retêm microplásticos por mais tempo que outros componentes da matriz de sedimentos ingerida. A ingestão microplástica por vermes Tubifex representa um risco significativo para transferência trófica e biomagnificação de microplásticos na cadeia alimentar aquática [24].

No lago Rawal, na capital do Paquistão, foi investigada, pela primeira vez, a presença e concentração de microplásticos nas águas superficiais e sedimentos do lago. A abundância de microplásticos em água de água e sedimentos foram de 0,142 itens/0,1 L e 1,04 itens/0,01 $\mathrm{kg}$, respectivamente. Nesse estudo, os resultados indicaram que as fibras e fragmentos eram os tipos mais dominantes de microplásticos. A incidência de microplásticos no lago Rawal afetam não apenas os organismos aquáticos e a cadeia alimentar, mas também pode ter impactos significativos na população das cidades vizinhas [25].

É interessante destacar um estudo pioneiro que relata a toxicidade comportamental induzida por MPs de polietileno em representantes de grupos de anfíbios. Physalaemus cuvieri tadpoles foram tratados por um corto período de tempo de 7 dias a uma concentração de MP's de polietileno de $60 \mathrm{mg} / \mathrm{L}$ e apresentaram problemas de locomoção, sintomas de efeito ansiogênico, bem como deficit de resposta defensiva anti-predatória quando expostos a predadores (Cyprinus carpio). A análise dos dados permitiu inferir até que ponto esses poluentes podem afetar os indivíduos e seus predadores naturais que vivem em áreas contaminadas [26]. O mesmo grupo de trabalho anterior mostrou que a bioacumulação de poluentes no fígado tadpoles estava correlacionada com diferentes alterações histopatológicas como dilatação dos vasos sanguíneos, infiltração, congestão, degeneração hidrópica, hipertrofia e hiperplasia, indicando a histopatotoxicidade dos MPs. Também, foram observadas alterações no tamanho dos núcleos dos hepatócitos, volume e forma induzidos pela exposição aos Mps, fato que evidenciou o efeito citotóxico desses poluentes [27].

\section{Efeitos sobre Zebrafish}

Existem trabalhos mostrando os efeitos dos poluentes orgânicos persistentes sobre o ambiente aquático, mas poucas evidências existem sobre os efeitos combinados da ingestão de poluentes orgânicos junto com os microplásticos. Zebrafish (Danio rerio) foi submetido durante 3 semanas a quatro tipos de alimentos diferentes (alimento não tratado; alimentos suplementados com microplásticos; alimentos suplementados com microplásticos a $2 \%$ com uma mistura de bifenilpoliclorado (PCB), retardantes de chama bromados (BFR), compostos perfluorados (PFCs) e metilmercúrio; e alimentos suplementados apenas com a mistura de contaminantes). Neste trabalho, os resultados indicaram que os microplásticos isoladamente não produzem efeitos relevantes ao peixe-zebra nas condições experimentais testadas, porém, o efeito combinado de microplásticos e contaminantes absorvidos modificou significativamente a homeostase de seus órgãos de uma maneira maior do que apenas com os contaminantes [28].

Os microplásticos são frequentemente consumidos por organismos marinhos por apresentarem tamanho semelhante aos seus itens alimentares, sendo potencialmente tóxicos e prejudiciais [29]. Foi observada a bioacumulação e toxicidade dos microplásticos nos organismos do peixe-zebra expostos a três formas de MPs (miçangas, fragmentos e fibras). Obtiveram como resultado o acúmulo dependente da forma na ordem de fibras $(8,0 \mathrm{mg} / \mathrm{mg})>$ fragmentos $(1,7 \mathrm{mg} / \mathrm{mg})>$ miçangas $(0,5 \mathrm{mg} / \mathrm{mg})$. Esses detritos mostraram efeitos negativos no intestino dos peixes, como danos a mucosa e elevação da permeabilidade, inflamação e interrupção do metabolismo, sendo o microplástico na forma de fibra o maior responsável pela severa toxicidade intestinal dos animais; além desses efeitos, foi visualizada a indução de disbiose da microbiota intestinal. É Importante destacar que, independentemente da forma microplástica capaz de causar impactos negativos, as outras formas não devem ser ignoradas na avaliação dos riscos à saúde ocasionados pelos microplásticos [30].

\section{Efeitos dos microplásticos no solo}

Até agora os impactos causados por microplásticos nos ecossistemas do solo, especialmente de terras agrícolas, apesar de serem reconhecidos como um grande reservatório de microplásticos. Diante disso, foi avaliada a resposta biofísica do solo usando diferentes tipos de microplásticos adicionados ao solo que continha a Aporrectodea rosea 
(minhoca de ponta rosada) endógena e plantada com Lolium perenne (azevém perene), como polietileno de alta densidade convencional (HDPE), fibras microplásticas de vestuário e ácido polilático biodegradável (PLA). Os resultados mostraram que menos sementes germinam quando expostas às fibras ou microplásticos PLA; houve também uma diminuição no $\mathrm{pH}$ do solo com a presença de HDPE; além disso foi reduzida consideravelmente a biomassa de A. rosea exposta ao HDPE quando comparada às amostras controle [31].

Os distúrbios das relações vitais entre o solo e a água, somado à função microbiana e a estrutura do solo foram estudadas por Machado et al (2018). Expuseram um solo argiloso a concentrações nominais ambientalmente relevantes (podendo chegar até $2 \%$ ) de quatro tipos de microplásticos comuns (fibras de poliéster, fibras poliacrílicas, contas de poliamida e fragmentos de polietileno), por um período de 5 semanas. Após esse tempo, mediu-se a capacidade de retenção de água, a densidade aparente, atividade microbiana, a condutividade hidráulica e a agregação do solo. Os resultados mostraram que os microplásticos afetaram a capacidade de retenção de água, a densidade aparente e a relação funcional entre a atividade microbiana e os agregados estáveis da água, sugerindo que os microplásticos são potencialmente grandes estressores antropogênicos de longo prazo e impulsionadores da mudança global nos ecossistemas terrestres [32].

\section{Efeitos químicos do plástico}

Vários aditivos e monômeros de plásticos são perigosos para a saúde humana e o meio ambiente. Por exemplo, di-(2-etil-hexil)-ftalato (DEHP) e bisfenol A, são tóxicos para a reprodução; cloreto de vinila, acrilonitrila, benzeno e 1,3-butadieno, são cancerígenos; formaldeído, acrilonitrila, diisocianato de tolueno (TDI) e metacrilato de metila, são alergênicos; benzeno, fenol e 1,3-butadieno, são mutagênicos; benzeno apresenta alta toxicidade crônica; fosgênio e TDI apresentam toxicidade aguda muito alta; éter pentabromodifenílico (PeBDE), acrilonitrila e TDI são ambientalmente perigosos com efeitos a longo prazo [33].

Sabe-se que trilhões de fragmentos de resíduo plástico flutua sobre o mar, mas apenas 1 a $2 \%$ entram nos oceanos anualmente. Para explicar o desaparecimento dos plásticos, Zhu et al. (2020) irradiou microplástico pós-consumo (polietileno, PE; polipropileno, PP; e poliestireno expandido, EPS) e obteve como resultado que a luz simulada pode remover resíduos de plástico do mar, além de fragmentar, oxidar e alterar a cor dos polímeros irradiados. No entanto, foi constatado que o carbono orgânico (DOC) é um dos principais subprodutos da fotodegradação plástica movida a luz solar e que esse DOC liberado foi amplamente utilizado por bactérias marinhas, havendo resultado em impactos mistos nos microrganismos oceânicos e nas redes alimentares que eles suportam [34].
Quando liberado no meio ambiente o microplástico sofre um tipo de envelhecimento e interage com substâncias, tais como contaminantes orgânicos, sendo essencial para avaliar o impacto do mesmo sobre o ambiente. Ao investigar os efeitos dos microplásticos de poliestireno acelerando seu envelhecimento por UV, análises físicas e química de partículas mostraram que o envelhecimento levou a uma oxidação superficial e uma pequena formação de microfissuras, concluindo que essa abordagem gerou estimativas aprimoradas das interações entre microplástico de poliestireno envelhecido e contaminantes orgânicos [35].

Os microplásticos podem apresentar efeitos negativos diretos, como também podem modificar a toxicidade e a biodisponibilidade dos poluentes. Foi testado durante uma semana o efeito combinado de três formulações químicas de glifosato (herbicidas) e dois tipos de microplásticos em Daphnia magna (um tipo de microcrustáceo), tendo como resultado a mudança na toxicidade das formulações, causando em algumas combinações até a mortalidade dos animais. E essas mudanças ocasionadas pelos microplásticos podem estar relacionadas com a conformação química dessas moléculas, e a interação das mesmas provoca efeitos duplos ruins [36].

O descarte do plástico no maio ambiente também contribui para a liberação de metais pesados, tais como mercúrio (Hg), normalmente utilizado na produção de cloro; trata-se do principal ingrediente do $\mathrm{PVC}$, do chumbo $(\mathrm{Pb})$ e do cádmio $(\mathrm{Cd})$, usados para a estabilização. Esses resíduos geralmente são liberados durante o uso e o descarte. Para avaliar os efeitos tóxicos nas espécies comerciais de peixes Lepidopus caudatus utilizou-se a expressão de vitelogenina e metalotioneínas; e em todas as amostras analisadas não foram encontrados microplásticos no trato gastrointestinal, mas sim uma grande quantidade de substâncias químicas, constatando-se, dessa forma, que os peixes podem reter esses metais pesados no organismo e repassá-los constantemente através da cadeia alimentar [37].

Para estudar o risco que podem causar à saúde humana foi avaliada, usando uma revisão sistemática de artigos publicados, a ingestão de microplásticos a partir da análise dos alimentos consumidos normalmente em uma dieta norte-americana, incluindo água potável, a qual representa aproximadamente $15 \%$ da ingestão calórica normal, e o potencial de inalação desse material; foi estimado que o consumo anual de microplásticos por pessoa varia de 39000 a 52000 partículas, dependendo da idade e do sexo; e esses números podem aumentar consideravelmente se as estimativas incluírem a inalação e a ingestão de água, chegando, nesse caso, a 90000 microplásticos consumidos anualmente por pessoa. Isso pode desencadear implicações para a saúde humana, representando assim um grande motivo de preocupação [38].

São evidentes as consequências do plástico no meio ambiente aquático, porém ele apresenta efeitos diretos sobre os seres vivos, incluindo o ser humano, devido ao 
consumo. Esses efeitos podem ser pela ingestão, ocasionado o estrangulamento, o aprisionamento ou a toxicidade. Com relação a essa última, o IARC (Agência Internacional de Pesquisa em Câncer) inclui diversos tipos plásticos, com seus componentes ou derivados que são causadores de câncer, como os derivados de PVC (policloreto de vinila) oi PS (poliestireno). Mesmo não havendo efeitos físicos e químicos direto em seres humanos, sabe-se que o plástico pode absorver contaminantes como PCBs (bifenilos policlorados), PAHs (hidrocarbonetos aromático policíclicos) ou DDT (diclorodifeniltricloroetano), aumentar relativamente o potencial efeito poluente, degradando-se em microplástico e causar impacto físico direto a partir da toxicidade, devido a incorporação de compostos químicos, tais como plastificantes, aditivos, metais pesados etc. [39].

\section{Conclusões}

Diante do que foi mostrado a respeito da contaminação dos ambientes aquáticos por meio do plástico, é necessário um monitoramento extenso, com amostragens sistemáticas em sedimentos e águas superficiais para entender melhor a origem dos insumos. A partir dos resultados apresentados em esta revisão, podemos observar dos elementos importantes a considerar: $\mathrm{O}$ primeiro é a constatação de que a maioria dos artigos publicados têm o seu foco nas águas marinhas em detrimento dos estudos realizados em água dolce, com o qual sugerimos e recomendamos, que mais esforços sejam dirigidos à pesquisa dos efeitos dos microplásticos sobre a água doce, considerando principalmente, que esta fonte de recursos naturais constituí uma mateira prima fundamental de diversas indústrias, como a pesqueira, e por suposto, um recurso primordial para a vida dos seres humanos. O segundo ponto a destacar é a observação, não quantitativa, da falta de uma metodologia específica para identificar e analisar a presença dos microplásticos em diversos ambientes naturais. Sem embargo, gostaríamos de sugerir, como ponto de partida, o seguimento dos métodos de laboratório de análises publicadas por Marine Debris Program, da National Oceanic and Atmospheric Administration [40] assim como também as metodologias publicadas pelo Joint Group of Experts on the Scientific Aspects of Marine Environmental Protection (GESAMP) [8]. Embora, essa falta de uma metodologia padronizada de análise esteja faltando e as pesquisas estar focadas nas águas marinhas, os resultados publicados mostram a gravidade que os microplásticos causam a diversos seres vivos, chegando como foi publicado, a afetar aos seres humanos também.

Embora ainda não tenham sido estabelecidas relações de causa e efeito conclusivas sobre a saúde humana, as pesquisas mostram diversos efeitos sobre a vida dos recursos hídricos, o que, consequentemente, pode acarretar inúmeros problemas no futuro. As substâncias e produtos chamados de poluentes emergentes, presentes no dia a dia da população, não são de simples identificação e não são removidos pelos sistemas convencionais de tratamento de esgoto existentes nos países [41].

Embora existam algumas campanhas lançadas para reduzir a quantidade de plásticos nos oceanos, são limitadas e dirigidas à consciência dos indivíduos. Em 2017, a Indonésia se comprometeu a reduzir o plástico lançado no mar; o Uruguai prometeu introduz imposto sobe os sacos plásticos e a Costa Rica sinalizou com a intenção de melhorar o gerenciamento de resíduos e a educação para reduzir o uso de sacolas. Foi proposto, naquele contexto, que, em 2020, sejam totalmente eliminadas as maiores fontes de plástico no mar: os microplásticos de cosméticos e as embalagens descartáveis. Mas, considerando os efeitos apresentados nesta revisão, o princípio de precaução deve ser priorizado por parte das autoridades sanitárias e ambientais dos diferentes países e organizações internacionais e não se limitar ao lançamento de campanhas publicitárias dirigidas à consciência das pessoas. Para finalizar, gostaríamos de destacar mais um dado preocupante publicado por Bletter et al [13], o qual mostra que a grande maioria das pesquisas sobre microplásticos em água doce é desenvolvida em países denominados do primeiro mundo, sendo, praticamente ignorados os problemas da poluição originada por los microplásticos sobre os recursos dos países em vias de desenvolvimento,

A evidência científica mostrando os macroproblemas que os microplásticos originam para o médio ambiente são mais que evidentes como para limitar o problema a uma questão simplesmente de curiosidade científica.

\section{Agradecimentos}

Agradecemos a todos os funcionários del DCN pelo o seu trabalho de apoio para todos os professores. LDGA agradece a la FCEYN de la Universidad de Buenos Aires pela formação de qualidade oferecida de forma gratuita e universal.

\section{Referencias}

1. A. R. A. Lima, M. F. Costa, and M. Barletta. Distribution patterns of microplastics within the plankton of a tropical estuary. Environ. Res., vol. 132, pp. 146-155, 2014.

2. T. Correa-Herrera, M. Barletta, A. R. A. Lima, L. F. Jiménez-Segura, and L. B. Arango-Sánchez. Spatial distribution and seasonality of ichthyoplankton and anthropogenic debris in a river delta in the Caribbean Sea. J. Fish Biol., vol. 90, no. 4, pp. 1356-1387, 2017.

3. M. S. Islam and M. Tanaka. Impacts of pollution on coastal and marine ecosystems including coastal and marine fisheries and approach for management: A review and synthesis. Mar. Pollut. Bull., vol. 48, no. 7-8, pp. 624-649, 2004.

4. R. Geyer, J. R. Jambeck, and K. L. Law. Production, use, and fate of all plastics ever made. Sci. Adv., vol. 3, no. 7, 
pp. 25-29, 2017.

5. L. C. M. Lebreton, J. Van Der Zwet, J. W. Damsteeg, B. Slat, A. Andrady, and J. Reisser. River plastic emissions to the world's oceans. Nat. Commun., vol. 8, pp. 1-10, 2017.

6. A. L. Andrady. Microplastics in the marine environment," Mar. Pollut. Bull., vol. 62, no. 8, pp. 1596-1605, 2011.

7. G. Caruso. Microplastics as vectors of contaminants. Mar. Pollut. Bull., vol. 146, no. April, pp. 921-924, 2019.

8. GESAMP. Sources, fate and effects of microplastics in the marine environment: a global assessment (Kershaw, P. J., ed.). (IMO/FAO/UNESCO-IOC/UNIDO/WMO/ IAEA/UN/UNEP/UNDP Joint Group of Experts on the Scientific Aspects of Marine Environmental Protection). Rep. Stud. GESAMP, vol. No. 90, p. 96 p., 2015.

9. J. P. G. L. Frias and R. Nash. Microplastics: Finding a consensus on the definition. 2019.

10. K. Duis and A. Coors. Microplastics in the aquatic and terrestrial environment: sources (with a specific focus on personal care products), fate and effects. Environ. Sci. Eur., vol. 28, no. 1, pp. 1-25, 2016.

11. D. G. de Carvalho and J. A. Baptista Neto. Microplastic pollution of the beaches of Guanabara Bay, Southeast Brazil. Ocean Coast. Manag., vol. 128, pp. 10-17, 2016.

12. P. Wu et al. Environmental occurrences, fate, and impacts of microplastics. Ecotoxicol. Environ. Saf., vol. 184, no. April, p. 109612, 2019.

13. M. C. M. Blettler, E. Abrial, F. R. Khan, N. Sivri, and L. A. Espinola. Freshwater plastic pollution: Recognizing research biases and identifying knowledge gaps. Water Res., vol. 143, pp. 416-424, 2018.

14. M. Bergmann et al. High Quantities of Microplastic in Arctic Deep-Sea Sediments from the HAUSGARTEN Observatory. Environ. Sci. Technol., vol. 51, no. 19, pp. 11000-11010, 2017.

15. B. Jovanović. Ingestion of microplastics by fish and its potential consequences from a physical perspective. Integr. Environ. Assess. Manag., vol. 13, no. 3, pp. 510-515, 2017.

16. S. M. Rodrigues et al. Microplastic contamination in an urban estuary: Abundance and distribution of microplastics and fish larvae in the Douro estuary. Sci. Total Environ., vol. 659, pp. 1071-1081, 2019.

17. v. Mouchi et al. Long-term aquaria study suggests species-specific responses of two cold-water corals to macro-and microplastics exposure. Environ. Pollut., vol. 253, pp. 322-329, 2019.

18. J. Reichert, J. Schellenberg, P. Schubert, and T. Wilke. Responses of reef building corals to microplastic exposure. Environ. Pollut., vol. 237, pp. 955-960, 2018.

19. [19] J. Reichert, A. L. Arnold, M. O. Hoogenboom, P. Schubert, and T. Wilke. Impacts of microplastics on growth and health of hermatypic corals are species-specific. Environ. Pollut., vol. 254, p. 113074, 2019.

20. R. Sussarellu et al. Oyster reproduction is affected by exposure to polystyrene microplastics. Proc. Natl. Acad.
Sci. U. S. A., vol. 113, no. 9, pp. 2430-2435, 2016.

21. M. Kazour, S. Jemaa, C. Issa, G. Khalaf, and R. Amara. Microplastics pollution along the Lebanese coast (Eastern Mediterranean Basin): Occurrence in surface water, sediments and biota samples. Sci. Total Environ., vol. 696, p. 133933, 2019.

22. M. Cole et al. Microplastic ingestion by zooplankton. Environ. Sci. Technol., vol. 47, no. 12, pp. 6646-6655, 2013.

23. A. A. Horton, A. Walton, D. J. Spurgeon, E. Lahive, and C. Svendsen. Microplastics in freshwater and terrestrial environments: Evaluating the current understanding to identify the knowledge gaps and future research priorities. Sci. Total Environ., vol. 586, pp. 127-141, 2017.

24. R. Hurley, J. C. Woodward, and J. J. Rothwell. Ingestion of Microplastics by Freshwater Tubifex Worms. Environ. Sci. Technol., vol. 51, no. 21, pp. 12844-12851, 2017.

25. T. Irfan, S. Khalid, M. Taneez, and M. Z. Hashmi. Plastic driven pollution in Pakistan: the first evidence of environmental exposure to microplastic in sediments and water of Rawal Lake. 2020.

26. A. P. da C. Araújo and G. Malafaia. Can short exposure to polyethylene microplastics change tadpoles' behavior? A study conducted with neotropical tadpole species belonging to order anura (Physalaemus cuvieri). J. Hazard. Mater., vol. 391, 2020.

27. A. P. da C. Araújo, A. R. Gomes, and G. Malafaia. Hepatotoxicity of pristine polyethylene microplastics in neotropical physalaemus cuvieri tadpoles (Fitzinger, 1826). J. Hazard. Mater., vol. 386, 2020.

28. S. Rainieri, N. Conlledo, B. K. Larsen, K. Granby, and A. Barranco. Combined effects of microplastics and chemical contaminants on the organ toxicity of zebrafish (Danio rerio). Environ. Res., vol. 162, no. December 2017, pp. 135-143, 2018.

29. S. Jinhui, X. Sudong, N. Yan, P. Xia, Q. Jiahao, and X. Yongjian. Effects of microplastics and attached heavy metals on growth, immunity, and heavy metal accumulation in the yellow seahorse, Hippocampus kuda Bleeker. Mar. Pollut. Bull., vol. 149, no. August, p. 110510, 2019.

30. R. Qiao et al. Accumulation of different shapes of microplastics initiates intestinal injury and gut microbiota dysbiosis in the gut of zebrafish. Chemosphere, vol. 236, p. 124334, 2019.

31. B. Boots, C. W. Russell, and D. S. Green. Effects of Microplastics in Soil Ecosystems: Above and Below Ground. Environ. Sci. Technol., vol. 53, no. 19, pp. 11496-11506, 2019.

32. A. A. De Souza MacHado et al. Impacts of Microplastics on the Soil Biophysical Environment. Environ. Sci. Technol., vol. 52, no. 17, pp. 9656-9665, 2018.

33. E. Commission. European Union Risk Assessment Report. 2002.

34. L. Zhu, S. Zhao, T. B. Bittar, A. Stubbins, and D. Li. Photochemical dissolution of buoyant microplastics to dissol- 
ved organic carbon: Rates and microbial impacts. J. Hazard. Mater., vol. 383, no. August 2019, p. 121065 , 2020.

35. T. Hüffer, A. K. Weniger, and T. Hofmann. Data on sorption of organic compounds by aged polystyrene microplastic particles. Data Br., vol. 18, pp. 474-479, 2018.

36. M. Zocchi and R. Sommaruga. Microplastics modify the toxicity of glyphosate on Daphnia magna. Sci. Total Environ., vol. 697, p. 134194, 2019.

37. A. Salvaggio et al. Biomarkers of Exposure to Chemical Contamination in the Commercial Fish Species Lepidopus caudatus (Euphrasen, 1788): A Particular Focus on Plastic Additives. Front. Physiol., vol. 10, no. July, pp. 1-13, 2019.
38. K. D. Cox, G. A. Covernton, H. L. Davies, J. F. Dower, F. Juanes, and S. E. Dudas. Human Consumption of Microplastics. Environ. Sci. Technol., vol. 53, no. 12, pp. 7068-7074, 2019.

39. C. B. Pastor and D. V. Agullól. Presencia de Microplásticos em Àguas y su Potencial Impacto em la Salud Pública. Rev. Esp. Salud Públic. vol. 93, pp. 1-10, 2019

40. N. Marine Debris Program. Laboratory Methods for the Analysis of Microplastics in the Marine Environment: Recommendations for quantifying synthetic particles in waters and sediments. No. July, 2015.

41. L. X. Nascimento and L. D. G. Alvarez. Contaminantes Orgánicos Emergentes: Impactos y Soluciones para la Salud Humana y el Medio Ambiente. Pp. 28-34, 2015. 